Number of pages: 16

Number of Figures: 13

Number of Tables: 0

Number of References: 18

\title{
Response of the seated human body to whole-body vertical vibration: biodynamic responses to mechanical shocks
}

\author{
Zhen Zhou and Michael J. Griffin \\ Human Factors Research Unit \\ Institute of Sound and Vibration Research \\ University of Southampton, \\ Southampton SO17 1BJ \\ England
}

Address for correspondence:

Professor Michael J Griffin

e-mail: M.J.Griffin@soton.ac.uk

Telephone: +44 (0)23 80592277

Facsimile: +44 (0)23 80592927 


\begin{abstract}
The biodynamic response of the seated human body has been investigated with 20 males exposed to upward and downward shocks at 13 fundamental frequencies (1 to $16 \mathrm{~Hz}$ ) and 18 magnitudes (up to $\pm 8.3 \mathrm{~ms}^{-2}$ ). For 1- and 2- degree-of-freedom models, the stiffness and damping coefficients were obtained by fitting seat acceleration waveforms predicted from the measured force to the measured seat acceleration waveform. Stiffness and damping coefficients were also obtained in the frequency domain with random vibration. The optimum stiffness and damping coefficients varied with the magnitude and the frequency of shocks. With both upward and downward shocks, the resonance frequency of the models decreased from 6.3 to $4 \mathrm{~Hz}$ as the vibration dose values of the shocks increased from 0.05 to $2.0 \mathrm{~ms}^{-1.75}$. The stiffness and damping obtained from responses to shocks were correlated with, and similar to, the stiffness and damping obtained with random vibration.
\end{abstract}

Key words: biodynamics; mechanical shocks; apparent mass; force; nonlinearity.

\title{
Practitioner summary
}

When modelling the dynamic response of the seated human body to vertical acceleration less than 1 $\mathrm{g}$, the relation between force and acceleration can be well represented by a single degree-of-freedom model although the optimum stiffness and damping depend on the magnitude and frequency of sinusoidal, random, or shock motion. 


\section{Introduction}

The biodynamic responses of the human body indicate how vibration is transmitted through the body and contribute to understanding of the effects of vibration on comfort, performance, and health. The transmission of vibration to the body through seating and other non-rigid structures is dependent on the biodynamic responses of the body.

The biodynamic responses of the human body to low frequency vibration are nonlinear. For example, with vertical excitation of the body the principal resonance frequency decreases if the magnitude of the vibration excitation is increased. This nonlinear softening effect has been found with both random and sinusoidal vibration (e.g., Hinz and Seidel, 1987; Fairley and Griffin, 1989; Matsumoto and Griffin, 2005; Toward and Griffin, 2010, 2011; Zhou and Griffin, 2014). With random vibration in the range 1 to $20 \mathrm{~Hz}$, Fairley and Griffin (1989) found that the mean apparent mass resonance frequencies of 8 seated subjects decreased from about $6 \mathrm{~Hz}$ to $4 \mathrm{~Hz}$ as the vibration magnitude increased from 0.25 to $2.0 \mathrm{~ms}^{-2}$ r.m.s. The frequency of a second resonance in the vertical apparent mass in the frequency range 8 to $12 \mathrm{~Hz}$ has also been observed to reduce as the magnitude of vibration excitation increases (e.g., Fairley and Griffin, 1989; Mansfield and Griffin, 2000).

With sinusoidal vibration (13 frequencies from 1 to $16 \mathrm{~Hz}$ at five magnitudes from 0.1 to $1.6 \mathrm{~ms}^{-2}$ r.m.s.) and with random vibration ( 1 to $16 \mathrm{~Hz}$ ) at the same magnitudes, the dependence of the apparent mass of the seated human body on the frequency, the magnitude, and the waveform of vertical vibration has been measured in 20 males and 20 females (Zhou and Griffin, 2014). The apparent mass was similar with random and sinusoidal vibration: with increasing magnitude of vibration, the resonance frequency decreased from 6.5 to $4.5 \mathrm{~Hz}$. This change in biodynamic response with increasing magnitude of vibration (i.e., the nonlinearity) depended on the frequency of the vibration excitation. Males and females had similar apparent mass (after adjusting for subject weight) and a similar principal resonance frequency with both random and sinusoidal vibration.

The apparent mass of the body has mostly been determined with random vibration, although it seems the frequency-dependence and magnitude-dependence of the apparent mass are similar with random and sinusoidal vibration. Few studies have investigated biodynamic responses to mechanical shocks, but with vertical transients at $4.0,5.0,6.3$, or $8 \mathrm{~Hz}$, the nominal apparent mass of the seated body was observed to decrease with increasing magnitude of the transient excitation (Matsumoto and Griffin, 2005). The limited investigation of human responses to shock restricts the modelling of biodynamic responses to shocks that are associated with discomfort and injury,

Although the body is a nonlinear multi-degree of freedom system, simple linear mass-spring-damper models provide surprisingly accurate representations of the modulus and phase of the vertical apparent mass of the seated human body exposed to random vibration (e.g., Fairley and Griffin, 1989; Toward and Griffin, 2009; Zhou and Griffin, 2014), and the addition of a second degree-of-freedom provides only a small improvement (Wei and Griffin, 1998). Such models have been developed to 
also represent the fore-and-aft forces on a seat during vertical excitation, by the addition of a rotational degree-of-freedom to represent the rotation of body segments during vertical excitation (e.g., Matsumoto and Griffin, 2001; Nawayseh and Griffin, 2009). Nonlinearity in the response of the human body implies that the response depends on the type of excitation (e.g., random or shock) and that a linear model representing the apparent mass of the body will require different parameters according to the magnitude of the excitation and the waveform of the excitation.

This study sought to identify what form of biodynamic model is required to represent the relation between force and acceleration when the seated human body is exposed to vertical shocks with peak accelerations less than $1 \mathrm{~g}$. For two alternative lumped-parameter time-domain models it was hypothesised that the parameters of the models would depend on both the magnitude of the shocks and the fundamental frequency of the shocks, reflecting the decrease in the resonance frequency of the apparent mass of the human body observed with increasing magnitudes of both sinusoidal and random vibration. There are no known previous studies of the apparent mass of the human body exposed to mechanical shocks using time domain methods.

\section{Experimental method and model description}

\subsection{Apparatus}

A 1-metre stroke vertical electrohydraulic vibrator generated vertical vibration of a flat rigid seat that was measured by an accelerometer (Silicon Designs 2260-002). A force platform (Kistler 9281B) mounted on the seat measured the force at the interface between the seat and the subject in the vertical. The effect of the mass of the top plate on the force platform was eliminated by subtracting the vertical acceleration multiplied by the mass of the top plate of the force platform (i.e., $31.5 \mathrm{~kg}$ ) from the measured vertical force in the time domain (i.e., mass cancellation). Sinusoidal vibration was generated by a Servotest Pulsar system and acquired using an HVLab data acquisition and analysis system (version 1.0) to a computer. The force and acceleration were acquired at 512 samples per second via $50-\mathrm{Hz}$ anti-aliasing filters.

Subjects sat on the top surface of the seat without making contact with a backrest (Figure 1). They rested their feet on a footrest that was attached to the vibrator table. The footrest was adjusted so that the upper surfaces of the upper legs were horizontal.

\section{FIGURE1 ABOUT HERE}

\subsection{Subjects}

Twenty male subjects, students at the University of Southampton, participated in the study. The median subject age was 24.5 years (range 22 - 33 years), mass $71.1 \mathrm{~kg}$ (range 48 - $107 \mathrm{~kg}$ ), stature $1.75 \mathrm{~m}$ (range $1.65-1.97 \mathrm{~m}$ ) and body mass index $23.1 \mathrm{~kg} / \mathrm{m}^{2}$ (range $17.6 \mathrm{~kg} / \mathrm{m}^{2}-27.6 \mathrm{~kg} / \mathrm{m}^{2}$ ).

During exposure to shocks, subjects were asked to close their eyes to prevent vision affecting their reaction to the motion. They were exposed to white acoustic noise at $65 \mathrm{~dB}(\mathrm{~A})$ via a pair of headphones. 
The experiment was approved by the Human Experimentation Safety and Ethics Committee of the Institute of Sound and Vibration Research at the University of Southampton. Informed consent to participate in the experiment was given by all subjects.

\subsection{Experimental design}

To obtain the shock acceleration waveforms, 11ㅡ-cycle sinusoidal waveforms were modulated by a half cycle sinusoid with a period three times longer than the period of the sinusoidal acceleration (Figure 2(a)).

\section{FIGURE 2 ABOUT HERE}

Subjects attended two sessions on each of two different days. In both sessions, subjects experienced 117 vertical shocks in the upward direction (an upward displacement as shown in Figure 2) and 117 vertical shocks in the downward direction in a completely random order over about 30 minutes. Different ranges of shock magnitude were presented on the two days (i.e., low or high), with the order balanced over the subjects.

Each of the $1 \frac{1}{2}$-cycle sinusoidal vibration waveforms had a frequency at one of the 13 preferred onethird octave centre frequencies in the range 1 to $16 \mathrm{~Hz}$. At each frequency, and for both the low magnitude and the high magnitude shocks, the shocks were presented at nine magnitudes, with the magnitudes adjusted to produce the same frequency-weighted vibration dose value, VDV, using the $W_{\mathrm{b}}$ frequency weighting in ISO 8041:2005. In the low magnitude session, the VDV at each frequency was in the range 0.05 to $0.315 \mathrm{~ms}^{-1.75}$ (with peak magnitudes up to $1.3 \mathrm{~ms}^{-2}$ r.m.s.). In the high magnitude session, the VDV at each frequency was in the range 0.315 to $2.0 \mathrm{~ms}^{-1.75}$ (with peak magnitudes up to $8.3 \mathrm{~ms}^{-2}$ r.m.s.). The ' $W_{\mathrm{b}}$ ' weighting was used so that, in accord with BS 6841 (1987) and previous research, shocks with the same VDV would be expected to produce similar discomfort.

After being exposed to all the shocks, subjects were exposed to random vertical vibration at five magnitudes $\left(0.1,0.2,0.4,0.8\right.$, and $1.6 \mathrm{~ms}^{-2}$ r.m.s.). The random vibration had approximately flat constant-bandwidth acceleration power spectra over the frequency range 1 to $16 \mathrm{~Hz}$. These $60-\mathrm{s}$ stimuli were presented in random order.

The experiment was also designed to measure the nonlinearity in the subjective responses to the vertical mechanical shocks. These subjective responses are reported separately (Zhou and Griffin, 2016).

\subsection{Model description}

A single-degree-of-freedom model and a two-degree-of-freedom model were used to represent the biodynamic response of human body exposed to mechanical shock (Figure 2(b)).

The motion equations for a single-degree-of-freedom model are:

$$
m_{1} \ddot{x_{1}}+c\left(\dot{x}_{1}-\dot{x}_{2}\right)+k\left(x_{1}-x_{2}\right)=F(t)
$$




$$
m_{2} \ddot{x}_{2}+c\left(\dot{x}_{2}-\dot{x}_{1}\right)+k\left(x_{2}-x_{1}\right)=0
$$

The motion equations for a two-degree-of-freedom model are:

$$
\begin{array}{r}
m \ddot{x}_{1}+c_{1}\left(\dot{x}-\dot{x}_{1}\right)+k\left(x-x_{1}\right)+c_{2}\left(\dot{x}-\dot{x}_{2}\right)+k_{2}\left(x-x_{2}\right)=F(t) \\
m_{1} \ddot{x_{1}}+c\left(\dot{x_{1}}-\dot{x}\right)+k\left(x_{1}-x\right)=0 \\
m_{2} \ddot{x}_{2}+c\left(\dot{x}_{2}-\dot{x}\right)+k\left(x_{2}-x\right)=0
\end{array}
$$

\subsection{Procedure to determine model parameters}

\subsubsection{Single-degree-of-freedom model}

The second-order ordinary differential equations ((1), (2)) were recast to a system of first order differential equations by introducing new variables (state space representation):

$$
y_{1}=x_{1}, \quad y_{2}=\dot{x}_{1}, \quad y_{3}=x_{2}, \quad y_{4}=\dot{x}_{2}
$$

The above motion equations were transferred to:

$$
\begin{gathered}
\dot{y}_{1}=y_{2} \\
\dot{y}_{2}=\frac{F(t)-c\left(y_{4}-y_{2}\right)-k\left(y_{3}-y_{1}\right)}{m_{1}} \\
\dot{y}_{3}=y_{4} \\
\dot{y}_{4}=\frac{c\left(y_{2}-y_{4}\right)+k\left(y_{1}-y_{3}\right)}{m_{2}}
\end{gathered}
$$

In the above equations, the force at the subject-seat interface is $F(t)$. The four model parameters $\left(m_{1}\right.$, $\left.m_{2}, c, k\right)$ are unknown. To minimise the risk of optimisation to inappropriate local minimum, the masses $m_{1}$ and $m_{2}$ were constrained to be $15 \%$ and $85 \%$ of the sitting masses of subjects, in accord with the findings of Wei and Griffin (1998). The sitting mass (i.e., $m_{1}+m_{2}$ ) was obtained from the measured vertical apparent mass of the subjects at $1 \mathrm{~Hz}$ during random vertical vibration (with a spectrum of 1 to $16 \mathrm{~Hz}$ at a magnitude of $0.4 \mathrm{~ms}^{-2}$ r.m.s.). The stiffness, $k$, and the damping coefficient, $c$, were determined by optimisation using the function (fmincon) provided in MATLAB (version R2010a). The interior point algorithm was used. The initial guesses and bounds of the stiffness and damping coefficient were determined from published data where the parameters $m_{1}, m_{2}, k$, and $c$ had been determined by fitting the model in the frequency domain to the apparent mass measured with 
random vibration (Figure 3 in Wei and Griffin, 1998). In 24 male subjects, they found optimum stiffness in the range 29,409 to $77,829 \mathrm{Nm}^{-1}$ and optimum damping coefficient in the range 675 to 2,345 $\mathrm{Nsm}^{-1}$. Considering the variability between subjects, the lower and upper bounds of the stiffness and damping coefficient in the present study were set to the range from 10,000 to $200,000 \mathrm{Nm}^{-1}$ and 100 to $10,000 \mathrm{Nsm}^{-1}$, respectively.

In each optimisation iteration, the above four first order differential equations were solved using a $4^{\text {th }}$ order Runge-Kutta method, and the velocity of $m_{1}$ (i.e., $y_{2}$ ) was obtained, then the acceleration of $m_{1}$ was calculated by differentiating the velocity of $m_{1}$. The model parameters were obtained by minimizing the difference between the fitted acceleration and the measured acceleration over the duration of the shock and the following one second, because the human body did not stop movement immediately after the end of a shock.

\subsubsection{Two-degree-of-freedom model}

Similar to the above single-degree-of-freedom model, the second-order differential equations ((3) to (5)) were recast to a system of first order differential equations. The seven model parameters $\left(m, m_{1}\right.$, $m_{2}, c_{1}, k_{1}, c_{2}, k_{2}$ ) are unknown. To minimise the risk of optimisation to inappropriate local minimum, the masses $m, m_{1}$ and $m_{2}$ were constrained to be $12 \%, 23 \%$ and $65 \%$ of the sitting masses of subjects, in accord with the findings of Wei and Griffin (1998). The values of the stiffness, $k_{1}, k_{2}$, and the damping coefficients, $c_{1}$ and $c_{2}$ were determined by optimisation as described above. The above two models are referred to as time-domain models.

\subsection{Apparent mass}

Based on the motion equations of the single degree-of-freedom model (Equations (1) and (2)), the nominal apparent mass of the human body exposed to shocks at each fundamental frequency can be obtained from:

$$
A M_{1 \mathrm{dof}}(i \omega)=\left[m_{1}+m_{2}\left(\frac{k+c_{1} i \omega}{k-m_{2} \omega^{2}+c i \omega}\right)\right]
$$

Then the apparent mass at each fundamental frequency can be calculated using the fitted parameters $(c, k)$ of the single degree-of-freedom model.

Similarly, with the motion equations of the two degree-of-freedom model (Equations (3) to (5)), the apparent mass can be obtained by:

$$
A M_{2 \mathrm{dof}}(i \omega)=\frac{D+E+(F+G) i}{A+B i}
$$

where:

$$
\begin{gathered}
A=k_{1} k_{2}-\omega^{2}\left(k_{1} m_{2}+k_{2} m_{1}\right)+m_{1} m_{2} \omega^{4}-c_{1} c_{2} \omega^{2} \\
B=\omega\left(k_{1} c_{2}+k_{2} c_{1}\right)-\omega^{3}\left(m_{1} c_{2}+m_{2} c_{1}\right)
\end{gathered}
$$




$$
\begin{gathered}
D=\left(m+m_{1}+m_{2}\right) k_{1} k_{2}-\left(m m_{2} k_{1}+m m_{1} k_{2}+m_{1} m_{2} k_{1}+m_{1} m_{2} k_{2}\right) \omega^{2} \\
E=m m_{1} m_{2} \omega^{4}-\left(m c_{1} c_{2}+m_{1} c_{1} c_{2}+m_{2} c_{1} c_{2}\right) \omega^{2} \\
F=\left(m+m_{1}+m_{2}\right)\left(k_{1} c_{2}+k_{2} c_{1}\right) \omega \\
G=-\left(m m_{1} c_{2}+m m_{2} c_{2}+m_{1} m_{2} c_{1}+m_{1} m_{2} c_{2}\right) \omega^{3}
\end{gathered}
$$

Then the apparent mass at each fundamental frequency can be calculated using the fitted parameters $\left(k_{1}, k_{2}, c_{1}, c_{2}\right)$ of the two degree-of-freedom model.

Each shock contained more than one frequency, but the frequency mentioned here is only the

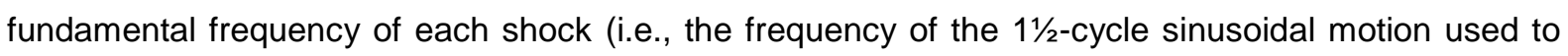
generate the shock). The apparent mass obtained here is therefore referred to as the 'nominal apparent mass' calculated for the various frequencies associated with that fundamental frequency.

With random vibration, the vertical apparent mass, $A M_{\text {random }}(\omega)$, was also calculated by the crossspectral density method:

$$
A M_{\text {random }}(\omega)=\frac{S_{i 0}(\omega)}{S_{i i}(\omega)}
$$

where $S_{i_{0}}(\omega)$ is the cross spectral density between the output (i.e., force) and the input excitation acceleration; $S_{\mathrm{ii}}(\omega)$ is the power spectral density of the input excitation acceleration at the vibrator platform. The calculation used a frequency resolution of $0.25 \mathrm{~Hz}$ and mass cancellation was performed in the time domain.

The stiffness and damping coefficient in the above single degree-of-freedom model were also obtained by fitting the model to the vertical apparent masses and phases measured with random vibration. The target error, $E(f)$, was calculated by summing the squared error in the modulus and the phase at each frequency between the measured data and the fitted response:

$$
E(f)=\sum_{N}\left[M_{\mathrm{m}}(f)-M_{\mathrm{s}}(f)\right]^{2}+\sum_{N}\left[P H_{\mathrm{m}}(f)-P H_{\mathrm{s}}(f)\right]^{2}
$$

where $N$ is the number of frequency points in the measured apparent mass (61 points for random vibration corresponding to the frequency range 1-16 Hz), $M_{\mathrm{m}}(f)$ and $P H_{\mathrm{m}}(f)$ are the apparent mass modulus and phase of the model at each frequency, and $M_{\mathrm{s}}(f)$ and $P H_{\mathrm{s}}(f)$ are the measured apparent mass modulus and phase. The constrained minimum error search command 'fmincon()' from the optimisation toolbox of MATLAB (version R2010a) was used for curve fitting. The constraints on the masses, and the initial guesses and bounds for the stiffness and damping coefficient were the same as described in Section 2.5 . 


\section{Results}

\subsection{Waveform of shock}

Examples of the measured input force, the measured output acceleration, and the fitted output acceleration when using a single degree-of-freedom model are shown for shocks having fundamental frequencies of $4 \mathrm{~Hz}$ and $16 \mathrm{~Hz}$ and the greatest magnitude (i.e., $2.0 \mathrm{~ms}^{-1.75}$ ) in Figure 3.

\section{FIGURE 3 ABOUT HERE}

The error, $\delta_{a}$, between the measured acceleration waveform, $a_{m}(t)$, and the fitted output acceleration waveform $a_{\mathrm{f}}(t)$, was examined using the following equation:

$$
\delta_{a}=\left(\frac{\int_{t+1}^{t 2}\left(a_{f}(t)-a_{m}(t)\right)^{2} d t}{\int_{t 1}^{t 2} \int\left(a_{f}(t)\right)^{2} d t}\right)^{3 / 2} \times 100 \%
$$

The integration was performed between $t_{1}$, the start time of the shock, and $t_{2}$, the end time of the shock. This measure of error is sensitive to errors in phase and not only errors in the magnitude or the shape of the acceleration waveform.

With both a single degree-of-freedom model and two degree-of-freedom model, the median error between the measured acceleration waveform and the fitted acceleration waveform (i.e., $\delta a)$ varied with the magnitude of the shock and the fundamental frequency of the shock (Figure 4). The median error tended to decrease as the magnitude of the shock increased but increase as the fundamental frequency increased, except for the high magnitude upward shocks with a fundamental frequency around $2 \mathrm{~Hz}$ where the acceleration peaks approached $1 \mathrm{~g}$. When the acceleration approached $1 \mathrm{~g}, \mathrm{a}$ sudden jump in the force was measured. This is assumed to have occurred because subjects left the seat (due to their downward acceleration under gravity being momentarily less than the downward acceleration of the vibrator) and then subsequently impacted with the seat. To develop the biodynamic models in this paper, the force was assumed to be the input, so the fitted acceleration did not provide a good fit to the measured acceleration waveform for these shocks with high magnitudes. For both models, the worst fit occurred at the higher frequencies where the phase difference between the measured acceleration and the fitted acceleration had a greater effect on the error.

\section{FIGURE 4 ABOUT HERE}

\subsection{Effect of shock magnitude and shock frequency on the stiffness, $k$}

The stiffness, $k$, of the equivalent single degree-of-freedom model as obtained by curve fitting varied with both the frequency and the magnitude of the shocks (Figure 5(a); the stiffness calculated for 1- $\mathrm{Hz}$ and $1.25-\mathrm{Hz}$ shocks are excluded because the human body was nearly rigid at these frequencies). As the fundamental frequency of shocks increased from 1.6 to $16 \mathrm{~Hz}$, the optimum stiffness generally increased. At frequencies greater than $2 \mathrm{~Hz}$, there were negative correlations between the magnitude of the shock and the median optimum stiffness over the 20 subjects at each frequency, for both upward shocks and downward shocks in both the lower and the higher range of magnitudes (Spearman rank correlation, $p<0.05$ ). 
The stiffnesses, $k_{1}$, and $k_{2}$, of the equivalent two degree-of-freedom model for upward shocks obtained by curve fitting are shown in Figure $5(b)$, and varied with both the frequency and the magnitude of the shocks. Similar to the single degree-of-freedom model, as the fundamental frequency of shocks increased from $1.6 \mathrm{~Hz}$ to $16 \mathrm{~Hz}$, the optimum value of both stiffnesses $\left(k_{1}\right.$ and $\left.k_{2}\right)$ generally increased. At frequencies greater than $1.6 \mathrm{~Hz}$, as the magnitude of the shocks increased, the stiffnesses mostly tended to reduce. The trend is more evident in the high magnitude shocks than in the low magnitude shocks, and also more consistent with $k_{2}$ than with $k_{1}$. Similar values and similar trends were found with downward shocks.

\section{FIGURE 5 ABOUT HERE}

\subsection{Effect of shock magnitude and shock frequency on the damping coefficient, $c$}

The damping coefficient, $c$, of the equivalent single degree-of-freedom model as obtained by curve fitting also varied with both the frequency and the magnitude of the shocks (Figure 6(a); the damping coefficient obtained at $1 \mathrm{~Hz}$ and $1.25 \mathrm{~Hz}$ is excluded because the human body is nearly rigid at these frequencies so there was insufficient relative motion within the body to obtain reliable estimates of the damping). The median optimum damping coefficient over the 20 subjects tended to decrease with increasing shock magnitude, especially with the higher range of shock magnitudes and with the higher frequencies of shock (Spearman rank correlation, $p<0.05$ ).

The damping coefficients, $c_{1}$, and $c_{2}$, of the equivalent two degree-of-freedom model for upward shocks as obtained by curve fitting are shown in Figure $6(b)$, and varied with both the frequency and the magnitude of the shocks. Similar to the stiffnesses $\left(k_{1}\right.$ and $\left.k_{2}\right)$, there are generally negative correlations between the damping coefficient and the magnitude of the shock. The trend is more evident in $c_{2}$ than $c_{1}$. Similar values and similar trends were found with downward shocks.

\section{FIGURE 6 ABOUT HERE}

\subsection{Nominal apparent mass during shock excitation}

With the obtained values of the stiffness, $k$, and the damping coefficient, $c$, the modulus and phase of the nominal apparent mass, $A M_{1 \text { dof }}$, of the body during shocks at each magnitude and each frequency were calculated using Equations (11).

To investigate the effect of shock magnitude on the nominal apparent mass, five magnitudes (i.e., $0.05,0.125,0.315,0.8$ and $2.0 \mathrm{~ms}^{-1.75}$ ) were chosen instead of all magnitudes used in the experiment. The greater magnitude difference was used to assist the illustration of the effect of vibration magnitude. As the magnitude of the shocks increased from $0.05 \mathrm{~ms}^{-1.75}$ to $2.0 \mathrm{~ms}^{-1.75} \mathrm{VDV}$, the median resonance frequency of the nominal apparent mass, $A M_{1 \mathrm{dof}}(\omega)$ (the nominal apparent mass measured assuming a single degree-of-freedom model) decreased from $6.3 \mathrm{~Hz}$ to $4 \mathrm{~Hz}$ with both downward and upward shocks ( $p<0.001$, Friedman, Figure 7). There was no significant difference in the resonance frequency of the nominal apparent mass between downward and upward shocks at any magnitude, except at $2.0 \mathrm{~ms}^{-1.75}$, where the resonance frequency was lower with the upward shocks $(p=0.028$, Wilcoxon) 


\section{FIGURE 7 ABOUT HERE}

Similar nominal apparent masses were found for $A M_{2 \text { dof }}(\omega)$ in the 2 degree-of-freedom model. The individual resonance frequencies were in the range 4 to $6 \mathrm{~Hz}$. As the magnitudes of the shocks increased from $0.05 \mathrm{~ms}^{-1.75}$ to $2.0 \mathrm{~ms}^{-1.75}$, the median resonance frequency of the nominal apparent mass $A M_{2 \text { dof }}$ also decreased from $6.3 \mathrm{~Hz}$ to $4 \mathrm{~Hz}$ with both downward and upward shocks $(p<0.001$, Friedman). There was no significant difference in the resonance frequency of the nominal apparent mass between the downward and upward shocks at any magnitude.

There were no significant differences in the resonance frequency obtained with $A M_{1 \text { dof }}$ and $A M_{2 \text { dof }}$ at any of the five magnitudes in either direction ( $p>0.05$, Wilcoxon). An example of the comparison between $A M_{1 \text { dof }}$ and $A M_{2 \text { dof }}$ is shown in Figure 8.

\section{FIGURE 8 ABOUT HERE}

\subsection{Apparent mass during random excitation}

The principal resonance in the vertical apparent mass during random vibration was about $5 \mathrm{~Hz}$, but depended on the magnitude of vibration and varied between subjects (from 4.75 to $8 \mathrm{~Hz}$ with $0.1 \mathrm{~ms}^{-2}$ r.m.s. and from 3.5 to $5.5 \mathrm{~Hz}$ with $1.6 \mathrm{~ms}^{-2}$ r.m.s.).

Similar to the apparent mass during shock excitation, the resonance frequency evident in the apparent mass during random excitation reduced as the magnitude of the excitation increased (Figure 9). As the magnitude of the random vibration increased from 0.1 to $1.6 \mathrm{~ms}^{-2}$ r.m.s., the median resonance frequency decreased from $6.5 \mathrm{~Hz}$ to $4.5 \mathrm{~Hz}(p<0.001$, Friedman).

\section{FIGURE 9 ABOUT HERE}

\subsection{Model parameters during random excitation}

With random vibration, the optimum stiffness and optimum damping coefficient of the single degreeof-freedom model (i.e., frequency-domain model) decreased with increasing magnitude of vibration $(p<0.001$, Friedman, Figure 10).

\section{FIGURE 10 ABOUT HERE}

With the single degree-of-freedom model, for each of the 20 subjects, the optimum stiffness and optimum damping coefficient obtained by fitting their measured response to $4-\mathrm{Hz}$ downward shocks at $0.315 \mathrm{~ms}^{-1.75}$ VDV (the greatest magnitude in the low magnitude downward session) were compared with the optimum stiffness and optimum damping coefficient fitted to their response to random vibration at $0.1 \mathrm{~ms}^{-2}$ r.m.s. (VDV of $0.324 \mathrm{~ms}^{-1.75}$ ). For both the stiffness and the damping coefficient, there were statistically significant positive correlations between values obtained during random vibration and the corresponding values obtained in response to shocks with fundamental frequencies greater than $2.5 \mathrm{~Hz}(p<0.05$; Spearman, Figure 11). For these motions having similar vibration dose values, both the stiffness and the damping coefficient were greater with random vibration than with shocks ( $p<0.05$, Wilcoxon). With other magnitudes of random vibration ( 0.628 and $1.256 \mathrm{~ms}^{-1.75} \mathrm{VDV}$ ) and other magnitudes of the shocks $\left(0.63\right.$ and $\left.1.25 \mathrm{~ms}^{-1.75} \mathrm{VDV}\right)$, the correlations between the 
optimum values for random vibration and the optimum values for shocks were similar, for both stiffness and damping coefficient.

\section{FIGURE 11 ABOUT HERE}

\subsection{Curve fitting of shock waveform with frequency-domain model}

The optimum stiffness and optimum damping coefficient obtained by fitting a single degree-offreedom model to the apparent mass measured with random vibration (with a magnitude of $0.1 \mathrm{~ms}^{-2}$ r.m.s.) were also used to predict the acceleration waveform for shocks in the low magnitude session.

The error between the measured acceleration waveform, $a_{m}(t)$, and the predicted acceleration waveform $a_{p}(t)$, $\delta a$, was examined using equation (21). Similar to Figure 4 , the median error between the measured acceleration waveform and the fitted acceleration waveform (i.e., סa) decreased as the magnitudes of the shocks increased but increased as the fundamental frequencies of the shocks increased (Figure 12). The median error was less than the median error obtained by fitting the single degree-of-freedom time-domain model. For example, with high magnitude downward shocks, the maximum median error was $20 \%$ at $16 \mathrm{~Hz}$ with the frequency domain model (Figure 12) but $30 \%$ at $16 \mathrm{~Hz}$ with the time domain model (Figure 4).

\section{FIGURE 12 ABOUT HERE}

\subsection{Associations between subject characteristics and biodynamic responses}

There was no clear pattern of correlations between the characteristics of the subjects (e.g., totalweight, stature, and body mass index) and the optimum stiffness with the two medium magnitudes of shock (i.e., $0.125 \mathrm{~ms}^{-1.75}$ in the low magnitude session, $0.8 \mathrm{~ms}^{-1.75}$ in the high magnitude session) at any frequency in any of the four sessions of the experiment. However, with both time-domain models, there were distinct patterns of statistically significant positive correlations between the above subject characteristics and the optimum damping coefficient for shocks having fundamental frequencies greater than $10 \mathrm{~Hz}$. There were no statistically significant correlations between the characteristics of the subjects (i.e., total-weight, stature, and body mass index) and the optimum stiffness or optimum damping coefficient of the model obtained for any of the five magnitudes of random vibration.

With five selected shock magnitudes (i.e., $0.05,0.125,0.315,0.8$, and $2 \mathrm{~ms}^{-1.75}$ ), the resonance frequency and the apparent mass at resonance were calculated from the stiffness and damping coefficient of a single degree-of-freedom model fitted to the shock response with both directions (upward and downward shocks). There were positive correlations between the physical characteristics of subjects (total-weight, stature, and body mass index) and both the resonance frequency of the apparent mass and the apparent mass at resonance frequency at all five magnitudes with both directions of shock (Kendall's $\tau_{\mathrm{b}} p<0.05$ ). With random vibration at all five magnitudes, there were also significant positive correlations between these three physical characteristics and the apparent mass at resonance. With random vibration, the resonance frequency of the apparent mass had significant positive correlations with the total weight and the body mass index of subjects, but only at low magnitudes (i.e., $0.1,0.2$, and $0.4 \mathrm{~ms}^{-2}$ r.m.s.). 


\section{Discussion}

\subsection{Proposed model}

The two time-domain models developed in this study provided reasonable fits to the measured time domain data, but with poorer fits at higher frequencies (Figure 4). The greater error at high frequencies seems to have been caused by increased phase difference between the fitted acceleration waveforms and the measured acceleration waveforms at higher frequencies. Example comparisons between waveforms are shown in Figure 13. The increased error around $2 \mathrm{~Hz}$ with the high magnitude upward shocks may have been caused by some form of nonlinearity when the peak magnitude approached $1 \mathrm{~g}$ and subjects were at risk of losing contact with the seat.

\section{FIGURE 13 ABOUT HERE}

As should be expected, the error between the measured and the fitted acceleration waveforms was less with the two degree-of-freedom model than with the single degree-of-freedom model (Figure 4). However, this is not sufficient to conclude that the two degree-of-freedom model is better than the single degree-of-freedom model. The optimum stiffness and optimum damping coefficient in the single degree-of-freedom model had more distinct patterns of statistically significant negative correlations with the magnitude of shocks. This suggests the single degree-of-freedom model might be more useful for simple representations of the nonlinearity of the body. With appropriate variations in model parameters, a single-degree of freedom model has previously been found to provide a good fit to the vertical apparent mass of the body over a range of sitting postures and a range of magnitudes of random vibration (0.125 to $1.6 \mathrm{~ms}^{-2}$ r.m.s.; Toward and Griffin, 2010). The less clear pattern in the current study with the greater number of variables in the two degree-of-freedom model suggests a more complex model may be required to represent biodynamic responses of the body exposed to a wide range of shock stimuli. Indeed, studies with random vibration excitation have shown that the biodynamic response of the body is much more complex than a single degree-of-freedom model (e.g., Kitazaki and Griffin, 1998; Matsumoto and Griffin, 2001; Nawayseh and Griffin, 2003).

At frequencies greater than $2.5 \mathrm{~Hz}$, the optimum stiffness and the optimum damping coefficient in a single degree-of-freedom model of the response of the body to mechanical shocks were positively correlated with the optimum stiffness and the optimum damping coefficient in the same model of response to random vibration (Figure 11). This shows subjects with greater stiffness and greater damping coefficient in response to random vibration tend also to have greater stiffness and greater damping coefficient in response to shocks. However, the stiffness and damping coefficient were greater during random vibration - possibly arising because the random vibration contained more high frequency components and more low frequency components than any shock. The random vibration had an approximately flat constant-bandwidth acceleration power spectra which means the higher frequency components had relatively higher magnitude in the random vibration than in the corresponding shocks, resulting in greater optimum stiffness (Figure 4(b)) and greater optimum damping coefficient (Figure 5(b)). This may have arisen because the optimum stiffness and optimum 
damping coefficient are dependent on both the magnitude and the frequency of vibration excitation, as shown elsewhere (Zhou and Griffin, 2014).

The acceleration waveforms measured in response to the shock forces tended to be more closely approximated by acceleration waveforms predicted by a single degree-of-freedom model optimised with random vibration in the frequency-domain than a model optimised with responses to shocks in the time-domain (compare top two figures in Figure 4 with top two figures in Figure 12). The reduced error at high frequencies in Figure 12 suggests optimisation in the frequency-domain provided a better definition of the phase between the force and acceleration waveforms. Whereas the time-domain model had 117 pairs of optimum stiffness and optimum damping, the frequency-domain model had only one value of stiffness and one value of damping to fit all 117 shock waveforms. It may be concluded that the frequency-domain approach using the biodynamic response to random vibration may be sufficient for some practical applications. However, the time-domain approach may assist understanding of the frequency-dependence and magnitude-dependence biodynamic responses of the human body exposed to mechanical shocks and other complex waveforms. The time-domain approach also allows the development of models of the nonlinearity in biodynamic responses of the body and the nonlinearity in seat suspension mechanisms.

\subsection{Nonlinearity in the vertical nominal apparent mass}

The study shows clear evidence of similar biodynamic nonlinearity in response to both mechanical shocks and random vibration. With shocks, as their magnitude increased from $0.05 \mathrm{~ms}^{-1.75}$ to $2.0 \mathrm{~ms}^{-}$ ${ }^{1.75}$, the median resonance frequency of the nominal apparent mass reduced from $6.3 \mathrm{~Hz}$ to $4 \mathrm{~Hz}$. With random vibration, as the magnitude of vibration increased from 0.1 to $1.6 \mathrm{~ms}^{-2}$ r.m.s., the median resonance frequency of the apparent mass reduced from $6.5 \mathrm{~Hz}$ to $4.5 \mathrm{~Hz}$. This 'softening' behaviour is similar to that found previously with random vibration (e.g., Fairley and Griffin, 1989), with sinusoidal vibration (e.g., Hinz and Seidel, 1987; Zhou and Griffin, 2014), and a few shocks similar to those in the present study (Matsumoto and Griffin, 2005).

The stiffness and the damping coefficients of both an optimum single degree-of-freedom model and an optimum two degree-of-freedom model reduced with increasing shock magnitude, except for the lowest frequencies of shock. It has been suggested that passive thixotropy of soft tissues, rather than geometric nonlinearity of the body or either voluntary or involuntary muscular activity, is the most likely primary cause of the nonlinearity in biodynamic responses to whole-body vibration (e.g., Matsumoto and Griffin, 2002; Huang and Griffin, 2008, 2009). The reduction in the stiffness and the damping coefficient with increasing magnitudes of shock is consistent with the thixotropic explanation.

With shocks of constant vibration dose value, the optimum stiffness increased greatly as the frequency increased above $3.15 \mathrm{~Hz}$ (Figure 5). It can be seen that the optimum stiffness was more dependent on the fundamental frequencies of the shocks than the magnitudes of the shocks. The relative motion between body parts is dependent on the frequency of the excitation as well as the magnitude of the excitation, and tends to decrease with increasing frequency of excitation. The reduction in the relative displacement with increasing frequency of excitation may contribute to the 
increased equivalent stiffness at high frequencies (the measured force decreased less than the relative displacement, so the equivalent stiffness increased), due to the nonlinearity of the body. However, the large increase in the optimum stiffness with increasing frequency is greater than would be expected if nonlinearity was the only explanation (see Figure 5). Part of the increase in equivalent stiffness with increasing frequency may have arisen because the model has been constrained to one or two degrees of freedom with fixed masses.

Previous studies have modelled the nonlinearity of the human body in the frequency domain with the Laplace transform. Since this is a linear operator, such models do not have nonlinear characteristics, merely different model parameters at different magnitudes. Nonlinear frequency-domain methods of modelling exist (e.g., Worden and Tomlinson, 2000), but their mathematical complexity is difficult to translate into physical meaning. The time-domain modelling method used in the present study (i.e., fitting model parameters directly using the motion equations) avoids these limitations. To model nonlinearities in biodynamic responses of the seated human body, explicit time-domain modelling may be simpler and more straightforward. Although the models used in the present study are linear, they may be developed into nonlinear models by adding nonlinear elements. This allows the development of a single model that represents the biodynamic response with varying magnitudes, frequencies, and waveforms.

The nonlinearity in the biodynamic response to vertical mechanical shocks found here can be expected to contribute to the nonlinearity observed in subjective responses to this type of mechanical shock (Zhou and Griffin, 2016). The time-domain modelling of the biodynamic responses employed here also offers a route to modelling and optimising the dynamic responses of seats to control the discomfort and injury potential associated with mechanical shocks.

\section{Conclusions}

Notwithstanding the nonlinearity and other complexities of the dynamic responses of the human body, the relation between the force and the acceleration at the input to a seated person excited by vertical mechanical shocks can be represented by either a single degree-of-freedom model or a two degreeof-freedom model in which the optimum stiffness and optimum damping vary with the magnitude of the shock and the fundamental frequency of the shock. The optimum stiffness and optimum damping obtained with a time-domain model of response to shocks are correlated with the optimum stiffness and optimum damping obtained with a frequency-domain model of response to random vibration. The frequency-domain model can also be used to predict the biodynamic responses to mechanical shocks. Similar to biodynamic responses to whole-body random vibration and whole-body sinusoidal vibration, the resonance frequency of the body evident in the equivalent apparent mass of the body during mechanical shocks reduces as the magnitude of the excitation increases.

\section{References}

Fairley, T.E. and Griffin, M.J., 1989. The apparent mass of the seated human body: Vertical vibration. Journal of Biomechanics, 22 (2), 81-94 
Hinz, B. and Seidel, H., 1987. The nonlinearity of the human body's dynamic response during sinusoidal whole body vibration. Industrial Health, 25, 169-181

Huang, Y. and Griffin, M.J., 2008. Nonlinear dual-axis biodynamic response of the semi-supine human body during vertical whole-body vibration. Journal of Sound and Vibration, 312 (1-2), 296-315.

Huang, Y. and Griffin, M.J., 2009. Nonlinearity in apparent mass and transmissibility of the supine human body during vertical whole-body vibration. Journal of Sound and Vibration, 324 (1-2), 429-452.

Kitazaki, S. and Griffin, M.J., 1998. Resonance behaviour of the seated human body and effects of posture. Journal of Biomechanics, 31, (1), 143-149.

Mansfield, N.J. and Griffin, M.J., 2000. Non-linearities in apparent mass and transmissibility during exposure to whole-body vertical vibration. Journal of Biomechanics, 33 (8), 933-941

Matsumoto, Y. and Griffin, M.J., 2001. Modelling the dynamic mechanisms associated with the principal resonance of the seated human body. Clinical Biomechanics, 16 (Sup.1), S31-S44

Matsumoto, Y. and Griffin, M.J., 2002. Effect of muscle tension on non-linearities in the apparent masses of seated subjects exposed to vertical whole-body vibration. Journal of Sound and Vibration, 253, (1), 77-92.

Matsumoto, Y. and Griffin, M.J., 2005. Nonlinear subjective and biodynamic responses to continuous and transient whole-body vibration in the vertical direction. Journal of Sound and Vibration, 287, 919937

Nawayseh, N. and Griffin, M.J., 2003. Non-linear dual-axis biodynamic response to vertical wholebody vibration. Journal of Sound and Vibration, 268

Nawayseh, N. and Griffin, M.J., 2009. A model of the vertical apparent mass and the fore-and-aft cross-axis apparent mass of the human body during vertical whole-body vibration. Journal of Sound and Vibration, 319 (1-2), 719-730

Toward, M.G.R. and Griffin, M.J., 2009. Apparent mass of the human body in the vertical direction: Effect of seat backrest. Journal of Sound and Vibration, 327, (3-5), 657-669.

Toward, M.G.R. and Griffin, M.J., 2010. A variable parameter single degree-of-freedom model for predicting the effects of sitting posture and vibration magnitude on the vertical apparent mass of the human body. Industrial Health, 48, 654-662.

Toward, M.G.R. and Griffin, M.J., 2011. Apparent mass of the human body in the vertical direction: Inter-subject variability. Journal of Sound and Vibration, 330, 827-841.

Wei, L. and Griffin, M.J., 1998. Mathematical models for the apparent mass of the seated human body exposed to vertical vibration. Journal of Sound and Vibration, 212 (5), 855-874

Worden, K. and Tomlinson, G.R., 2000. Nonlinearity in structural dynamics: Detection, identification and modelling. Taylor and Francis.

Zhou, Z. and Griffin, M.J., 2014. Response of the seated human body to whole-body vertical vibration: Biodynamic responses to sinusoidal and random vibration. Ergonomics, 57 (5), 693-713.

Zhou, Z. and Griffin, M.J., 2016. Response of the seated human body to whole-body vertical vibration: Discomfort caused by mechanical shocks. Submitted to Ergonomics. 


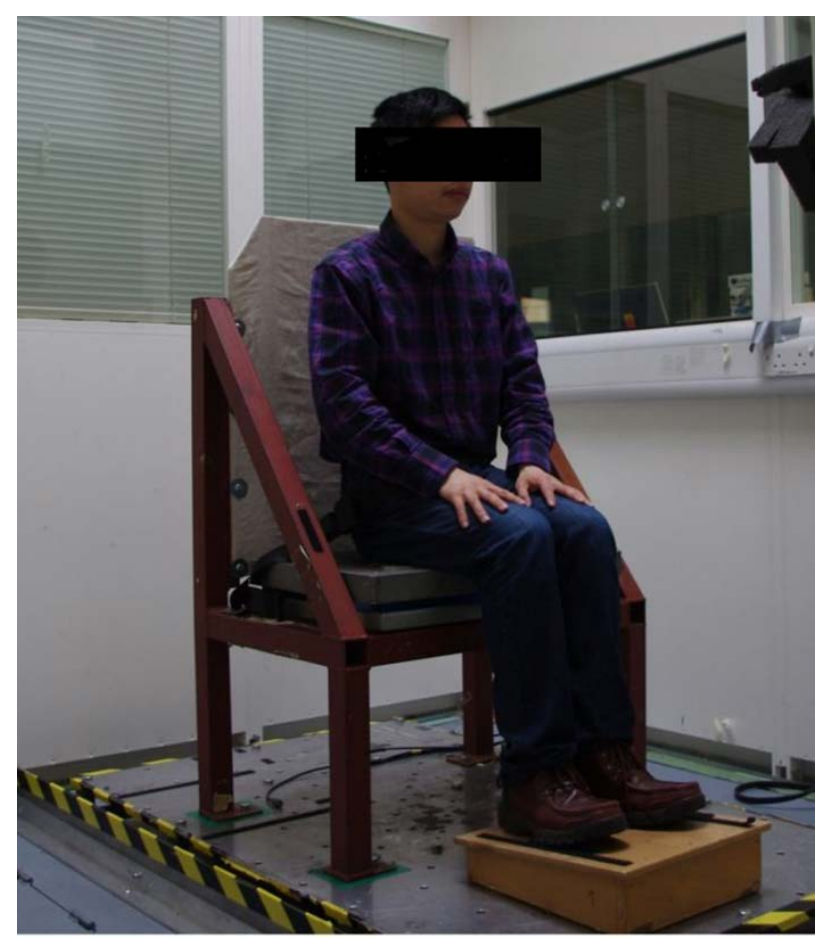

Figure 1 Experiment setup 


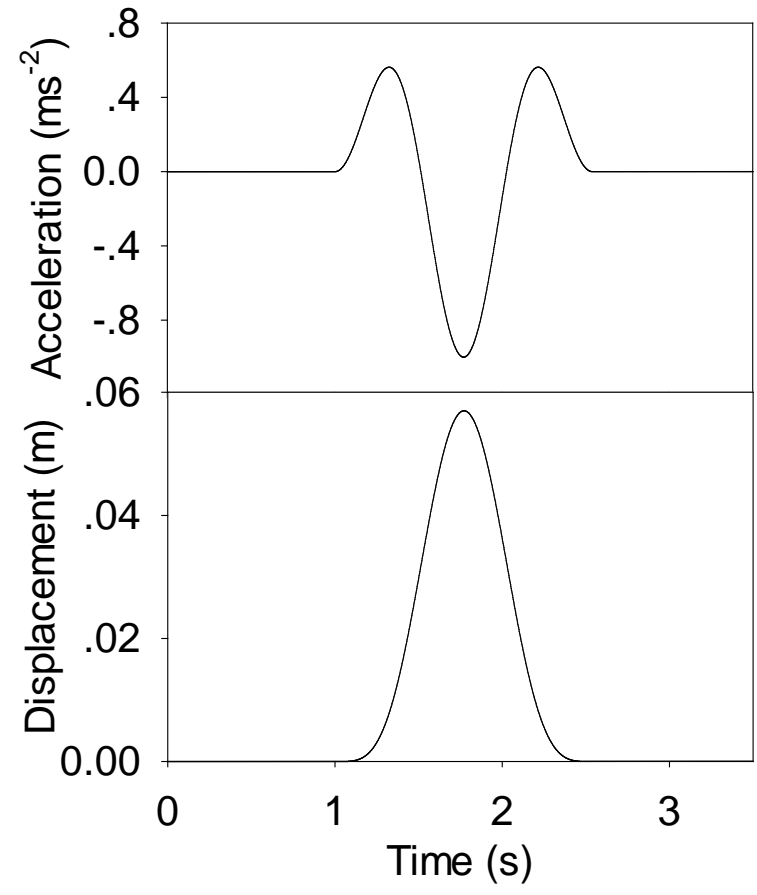

(a)

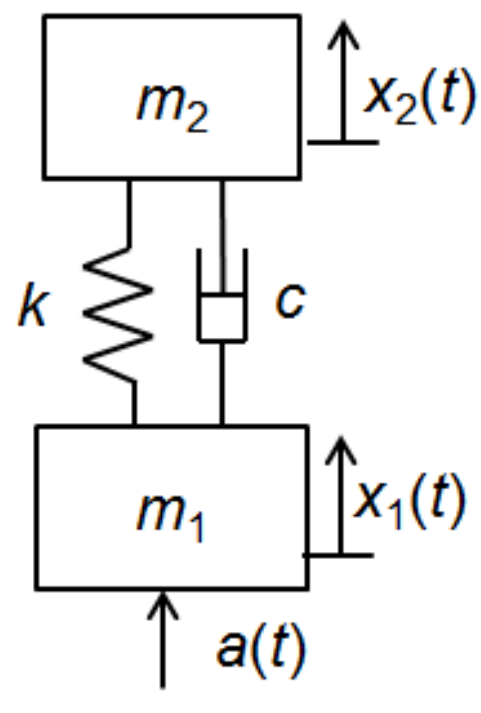

(b)

Figure 2 Example acceleration and displacement waveforms of the shocks (a), and single degreeof-freedom and two degree-of-freedom models used in the study (b). 

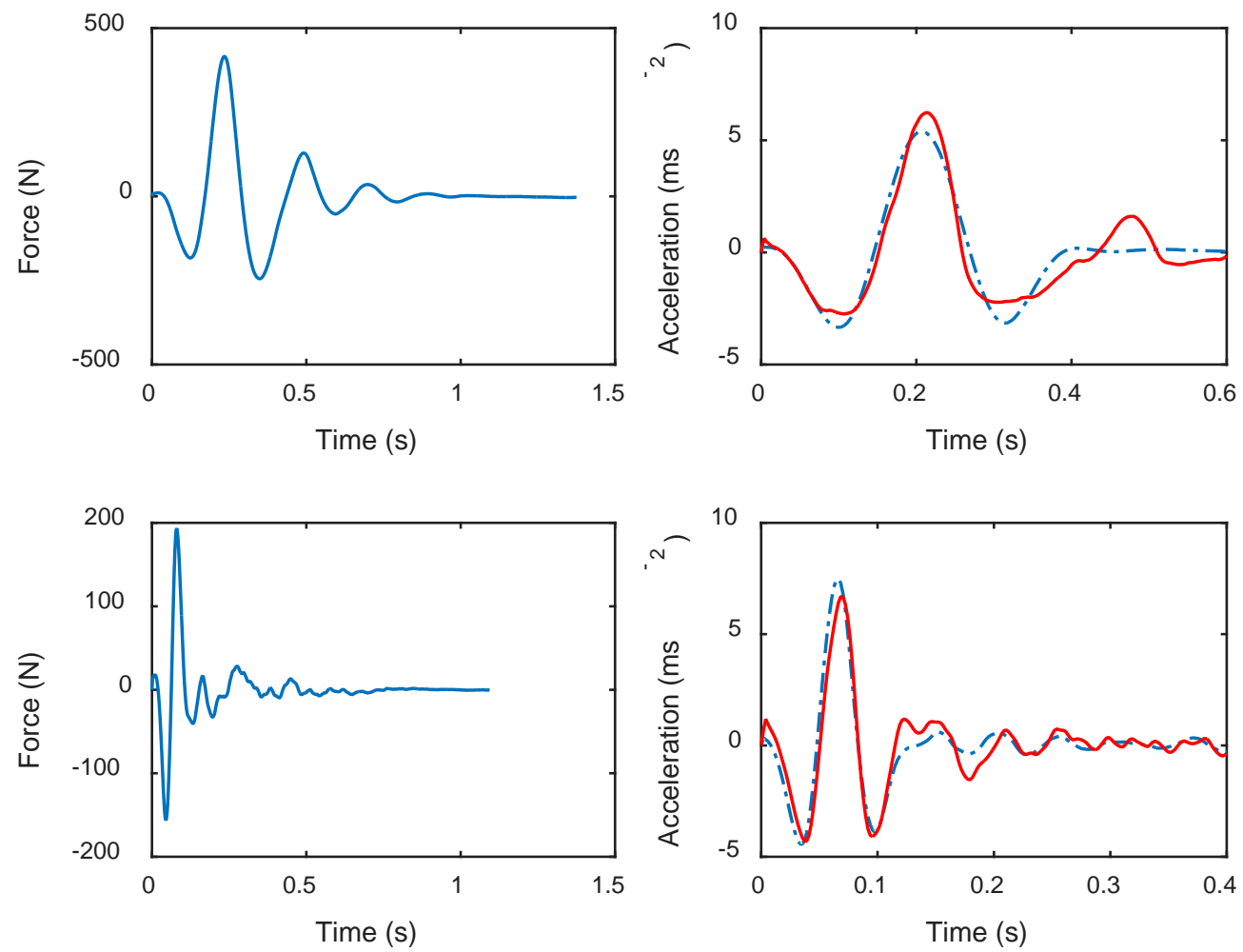

Figure 3 Examples of the measured input force, the measured output acceleration, and the predicted output acceleration waveforms for two shocks. Upper graphs: 4-Hz nominal frequency with a VDV of $2.0 \mathrm{~ms}^{-1.75}$; lower graphs: $16-\mathrm{Hz}$ nominal frequency with a VDV of $2.0 \mathrm{~ms}^{-1.75}$; left: measured input force waveforms; right: measured output acceleration waveforms $\left(\ldots_{-}\right)$and fitted output acceleration waveforms $({ } \quad$ ) for single degree-of-freedom models. 

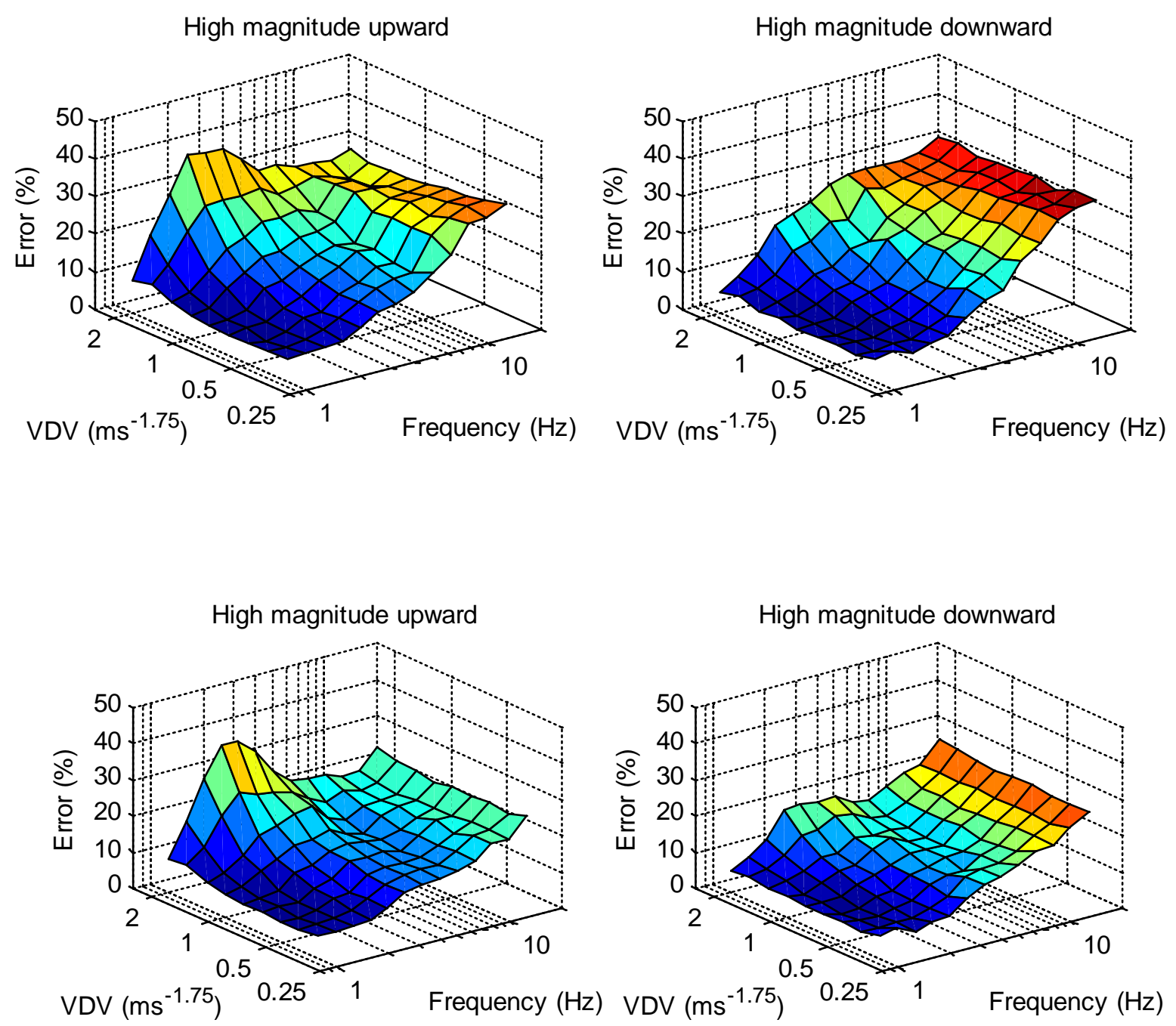

Figure 4 Median error between the measured acceleration waveforms and the fitted acceleration waveforms (i.e., $\delta a$ ) with the single degree-of-freedom model (upper figures) and the two degree-of-freedom model (lower figures) at each frequency with higher magnitude upward shocks (left figures) and higher magnitude downward shocks (right figures). Median values over 20 subjects at each of nine magnitudes of shock shown in Table 1. (Similar results were found with low magnitude upward and downward shocks.) 
High magnitude upward

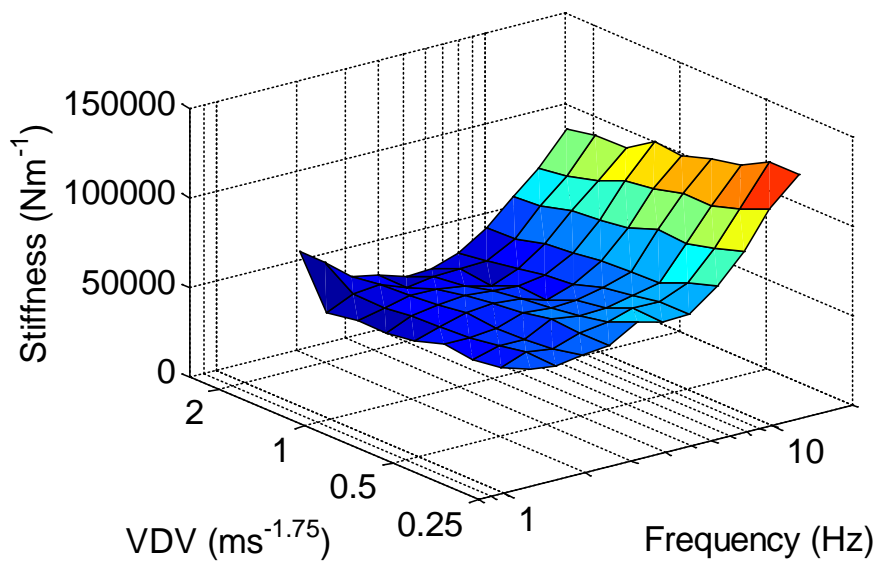

High magnitude downward

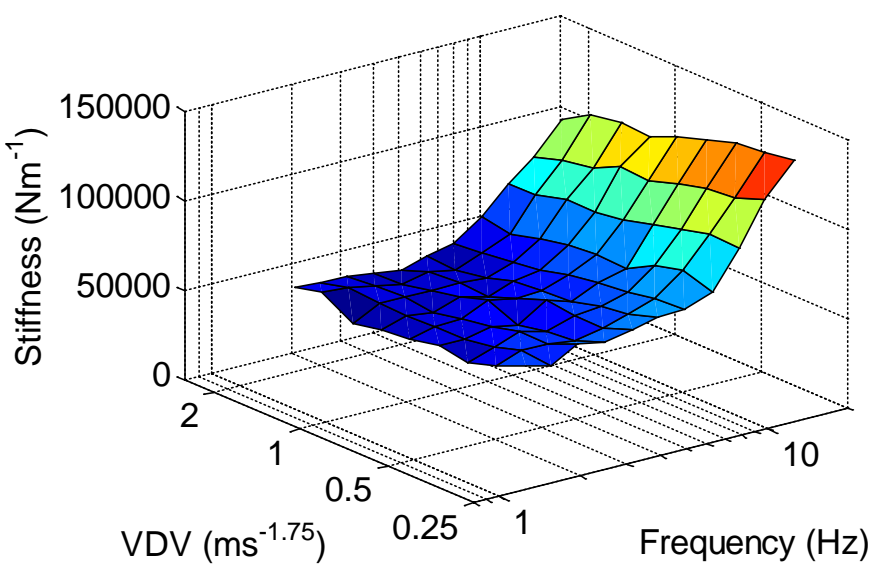

High magnitude upward

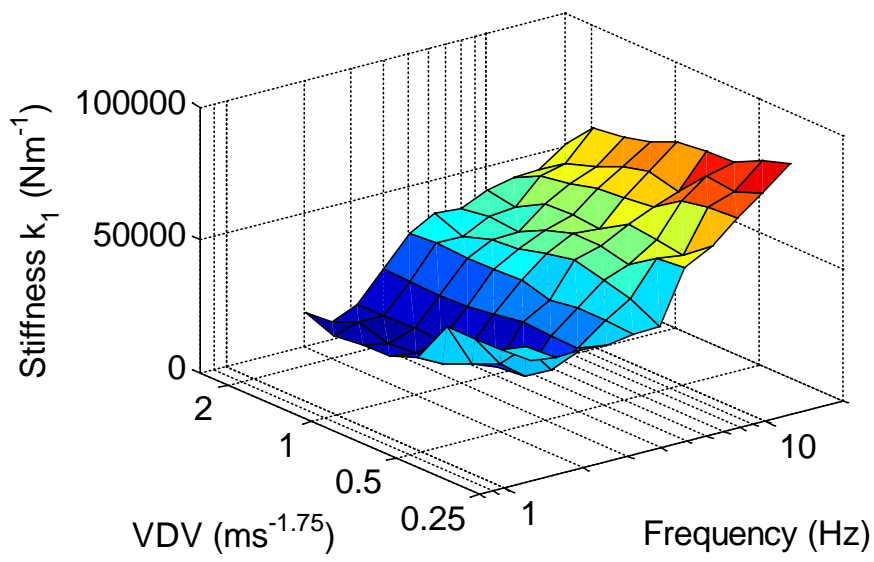

High magnitude upward

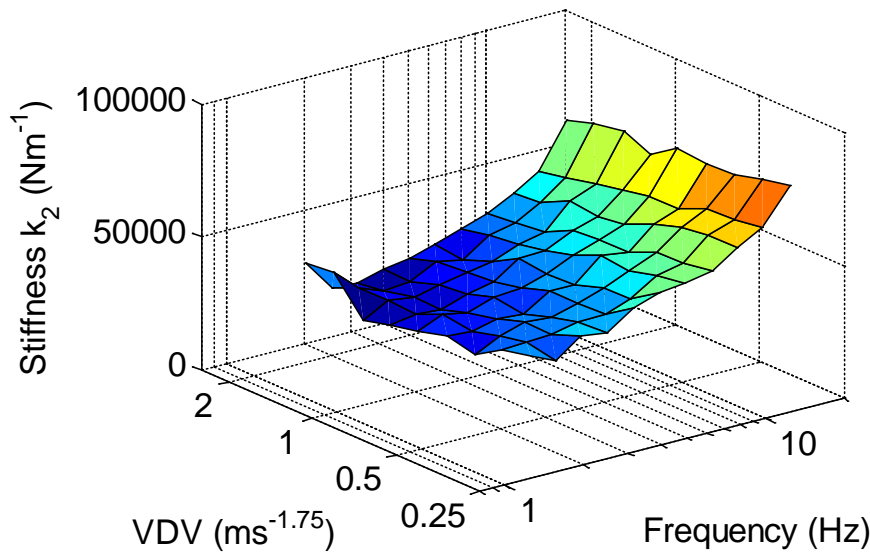

Figure 5 Upper figures: optimum stiffness, $k$, of a single degree-of-freedom model for each magnitude and nominal frequency of shock: high magnitude upward shocks (left figures) and high magnitude downward shocks (right figures); Lower figures: optimum stiffnesses, $k_{1}$ and $k_{2}$ of a two degree-of-freedom model for each magnitude and nominal frequency of shock: high magnitude upward shocks. Median values over 20 subjects. (Similar results with other shocks.) 

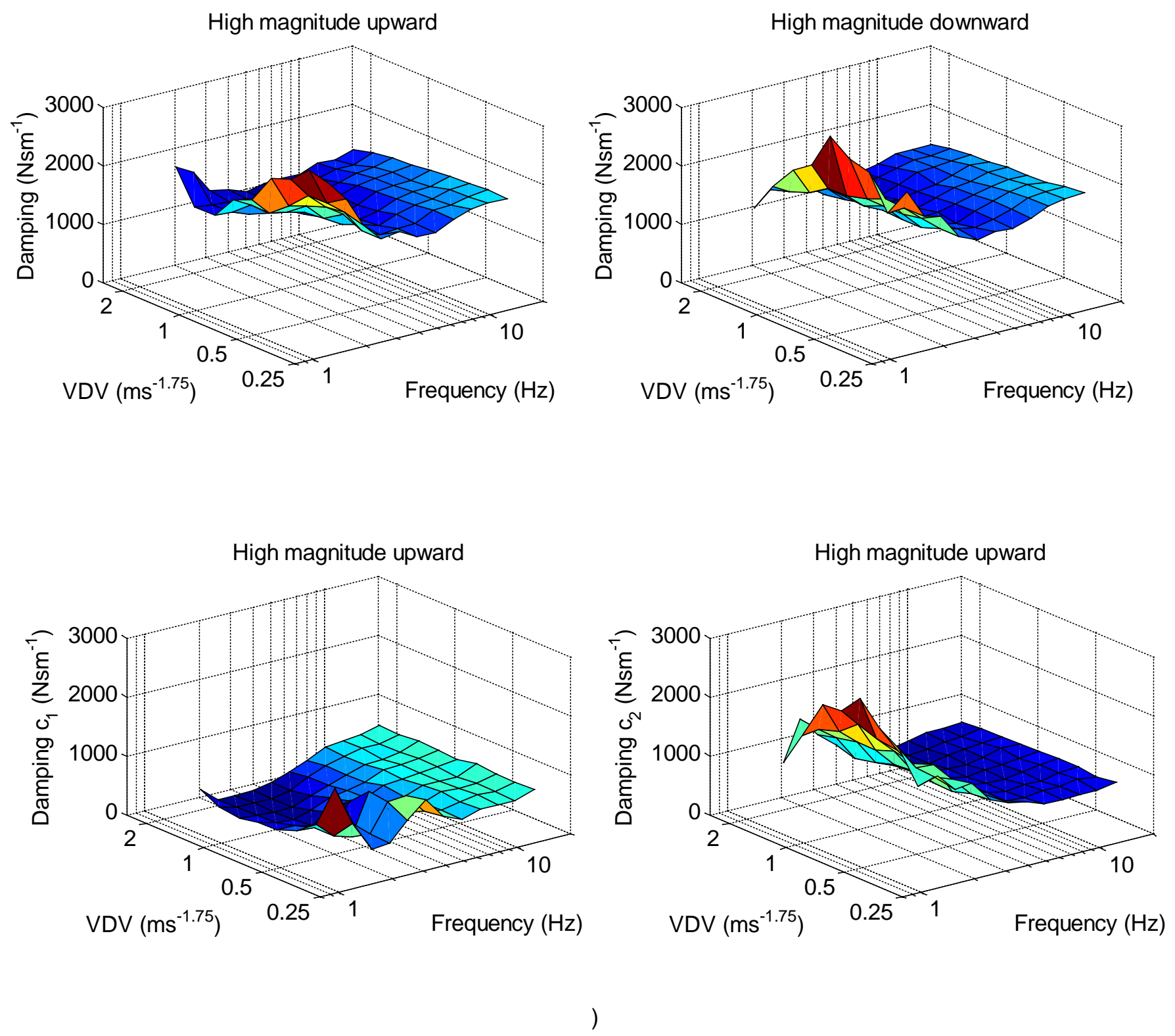

Figure 6 Upper figures: optimum damping, $c$, of single degree-of-freedom model for each magnitude and nominal frequency of shock: high magnitude upward shocks (left figures) and high magnitude downward shocks (right figures); Lower figures: Optimum damping, $c_{1}$ and $c_{2}$ of a two degree-of-freedom model for each magnitude and nominal frequency of shock: high magnitude upward shocks. Median values over 20 subjects. (Similar results were found with other shocks.) 


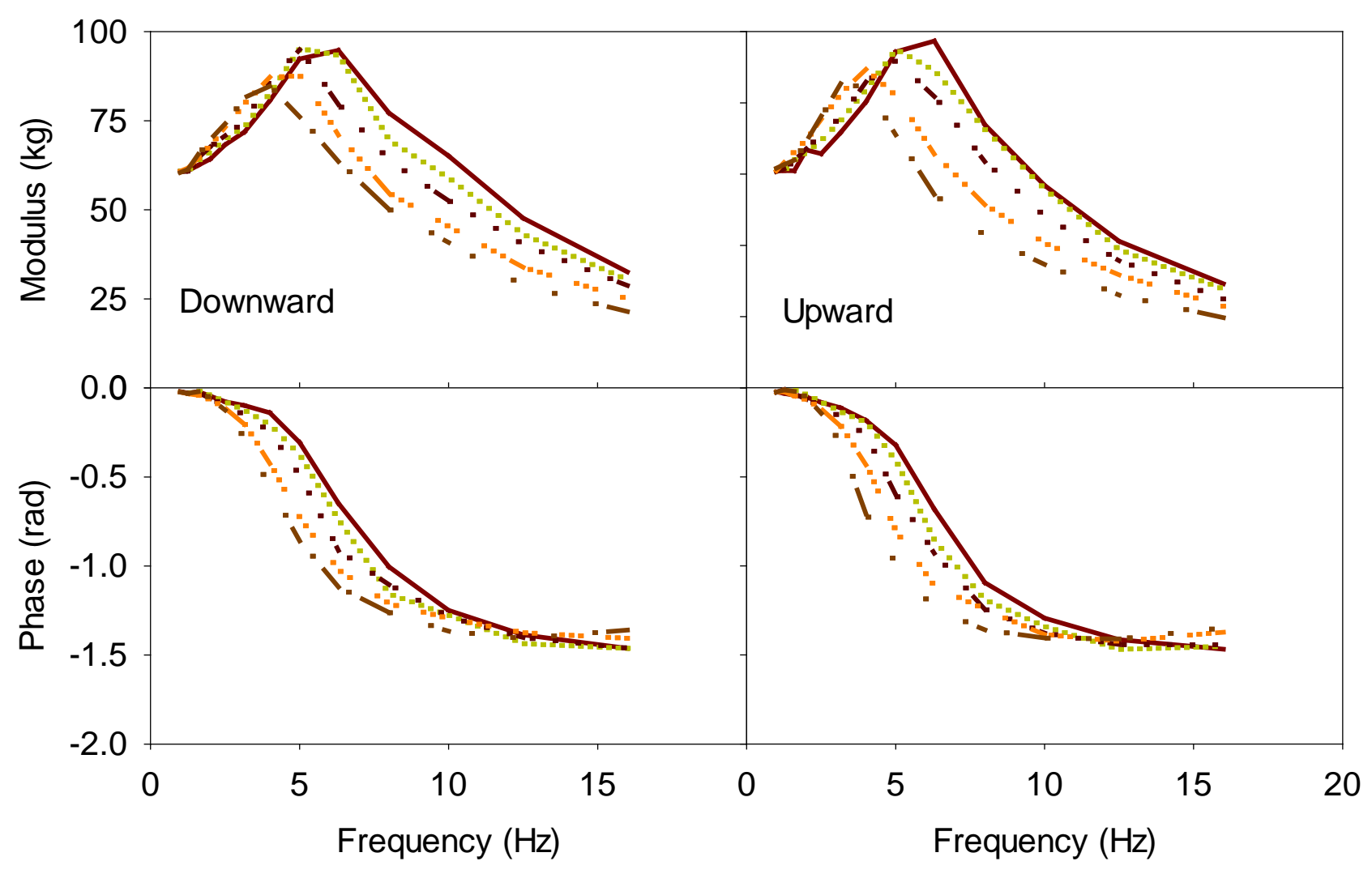

Figure 7 Median modulus and phase of the nominal apparent mass, $A M_{\text {sdof }}(\omega)$ (the nominal apparent mass measured assuming a single degree-of-freedom model), for subjects exposed to downward shocks (left figures) and upward shocks (right figures) at five magnitudes (一: $0.05 \mathrm{~ms}^{-1.75} ; \cdots: 0.125 \mathrm{~ms}^{-1.75} ;-\div: 0.315 \mathrm{~ms}^{-1.75} ;-\cdots-: 0.8 \mathrm{~ms}^{-1.75}$; $\left.\longrightarrow: 2.0 \mathrm{~ms}^{-1.75}\right)$. 


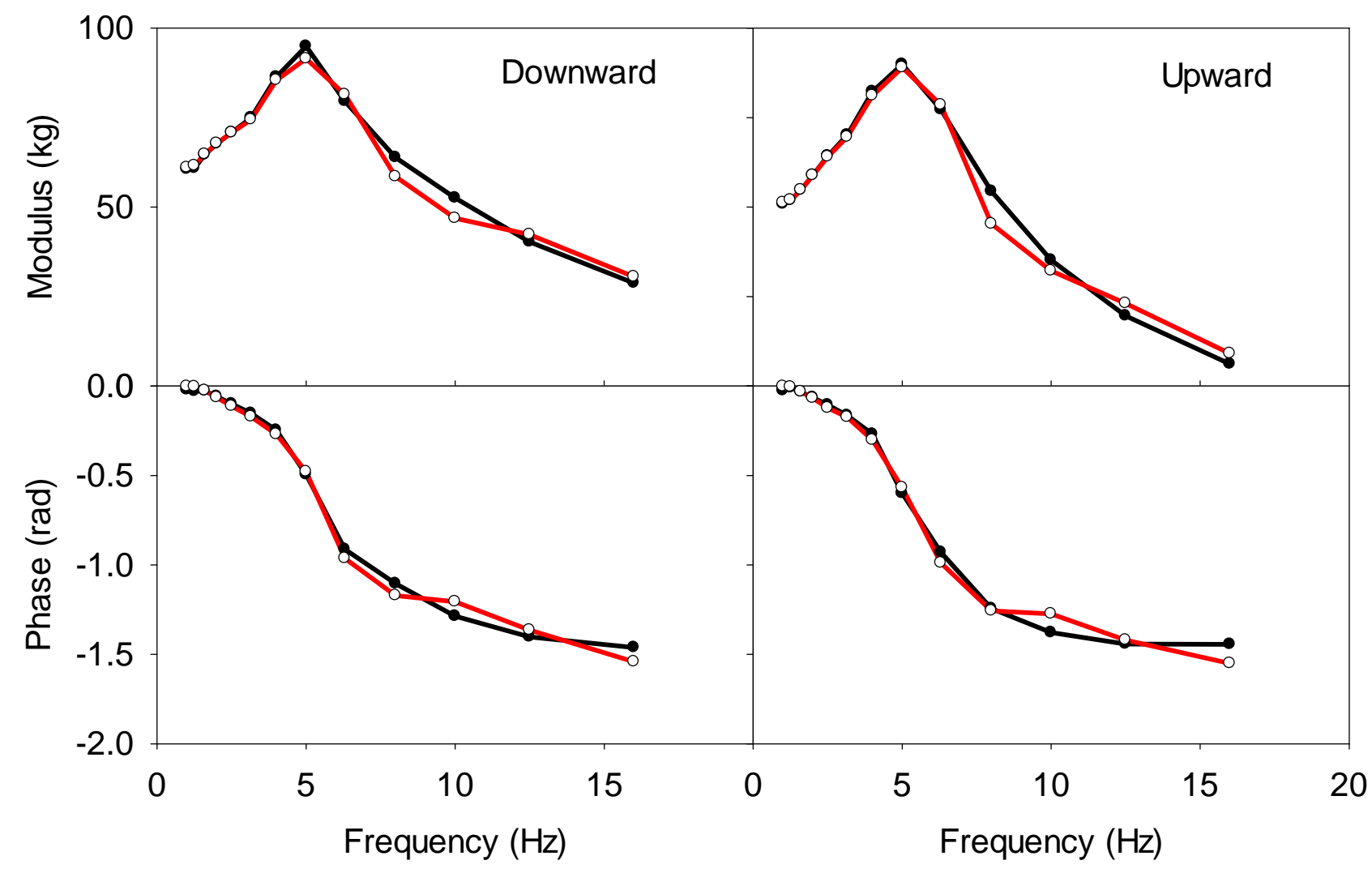

Figure 8 Example comparison of the modulus and phase of the apparent mass calculated from the single degree-offreedom model, $A M_{1 \mathrm{dof}}(\omega)(-\bullet-)$ and the two degree-of-freedom model, $A M_{2 \mathrm{dof}}(\omega)(-\circ-)$. The data are calculated from the responses of 20 subjects exposed to downward and upward shocks at $0.315 \mathrm{~ms}^{-1.75}$. 


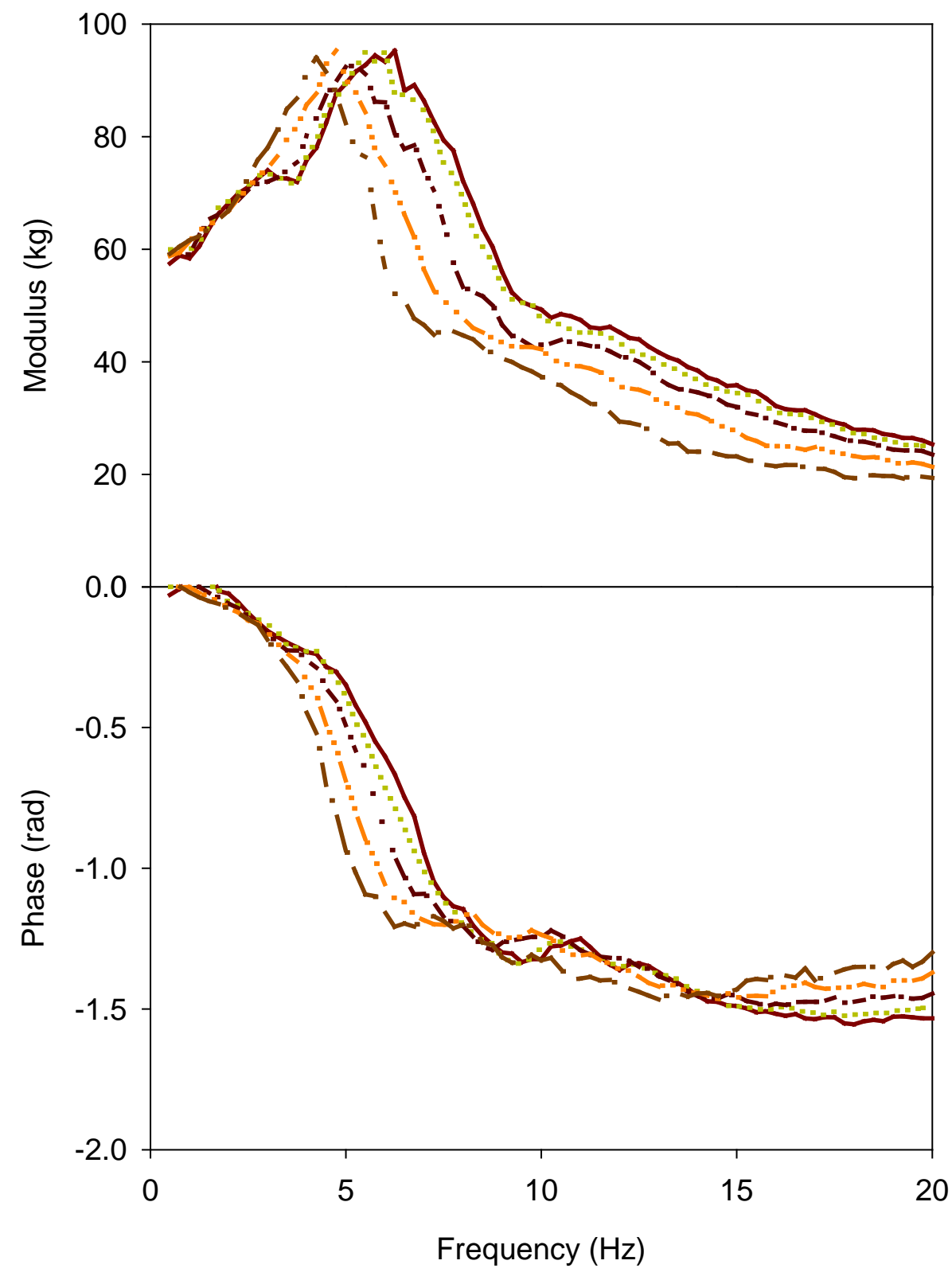

Figure 9 Median modulus and phase of the apparent mass of subjects exposed to vertical random vibration at five magnitudes (—: $0.1 \mathrm{~ms}^{-2}$ r.m.s.; $\cdots: 0.2 \mathrm{~ms}^{-2}$ r.m.s.; - -: $0.4 \mathrm{~ms}^{-2}$ r.m.s.; - - -: $0.8 \mathrm{~ms}^{-2}$ r.m.s.; ——: $1.6 \mathrm{~ms}^{-2}$ r.m.s.) 

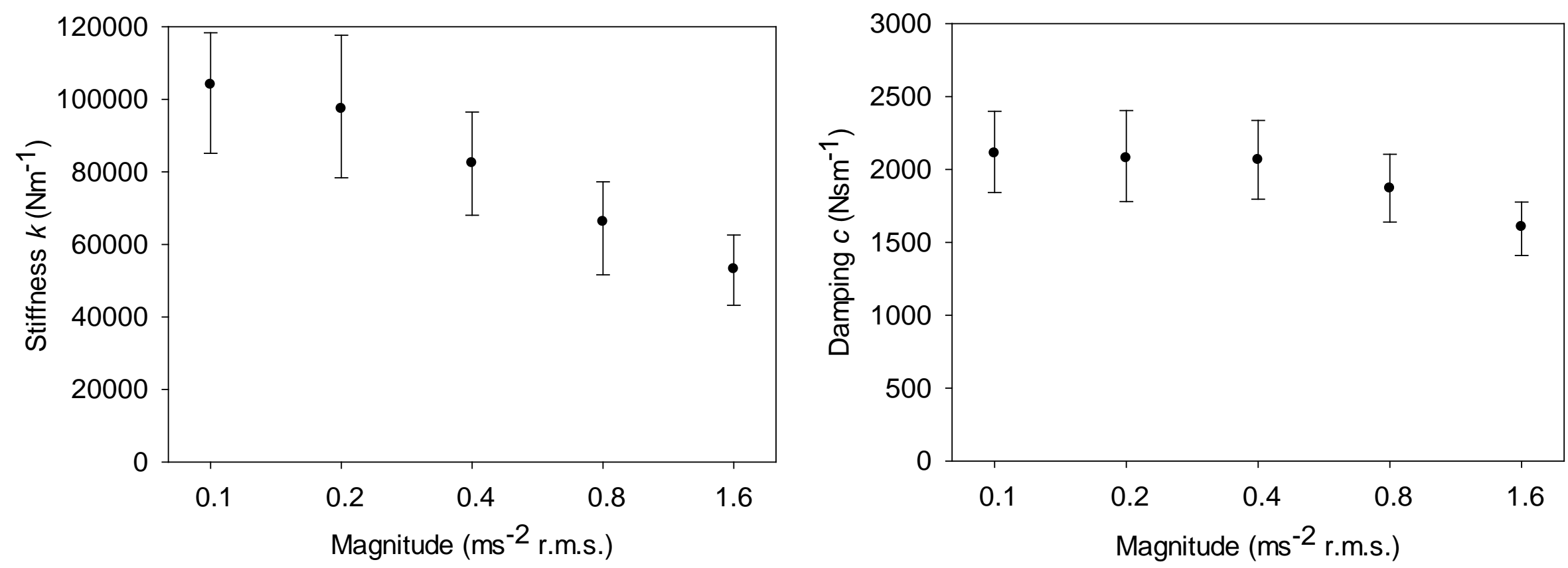

Figure 10 Median and inter-quarter range of optimum stiffness and optimum damping of the single degree-of-freedom model from five magnitudes of random vibration. 
Response of the seated human body to whole-body vertical vibration: biodynamic responses to mechanical shocks Zhou, Z. \& Griffin, M. J. 2017 In : Ergonomics. 60, 3, p. 333-346 14 p.
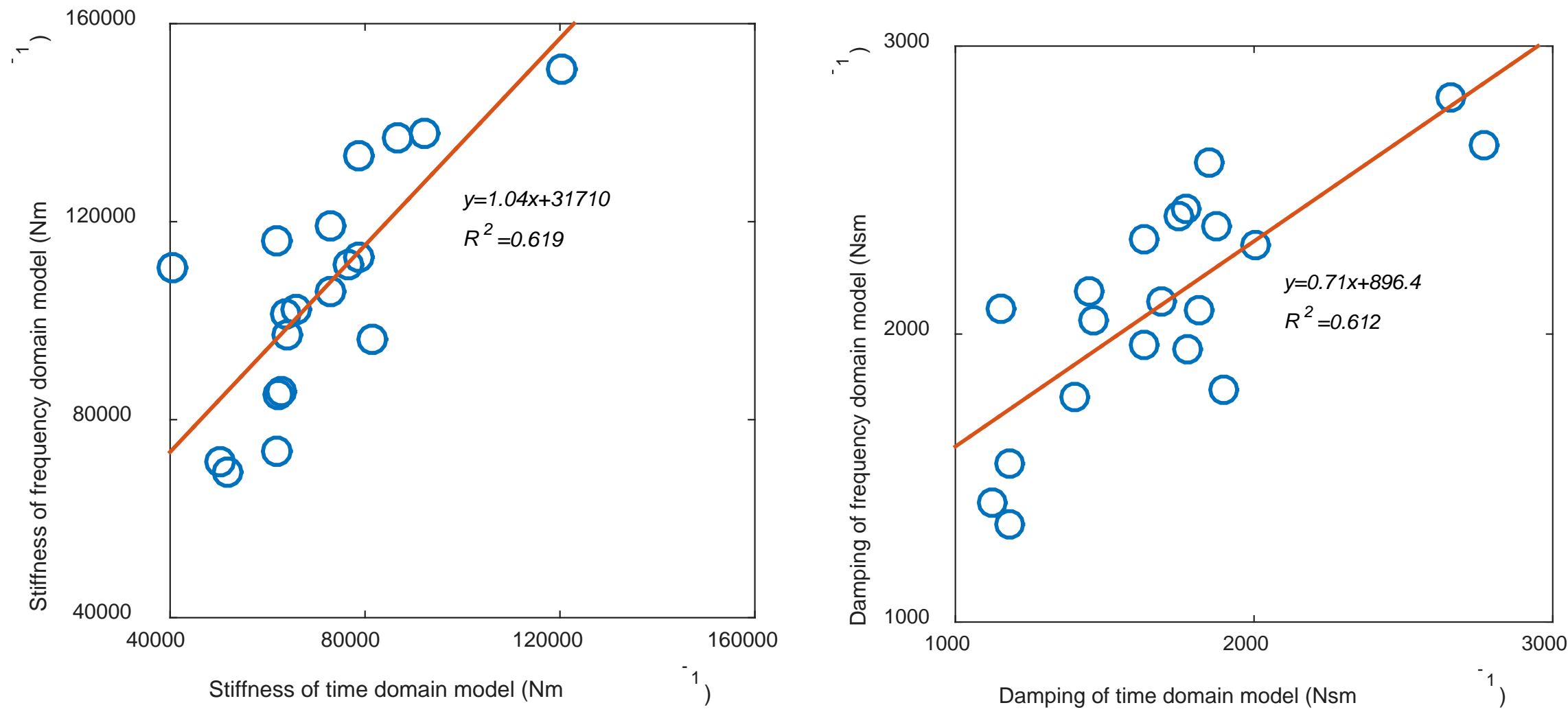

Figure 11 Example correlations between the optimum stiffness and optimum damping of a frequency-domain single degree-of-freedom model of response to random vibration (at $0.1 \mathrm{~ms}^{-2}$ r.m.s.; $0.324 \mathrm{~ms}^{-1.75}$ ) and the corresponding optimum parameters of a time-domain single degree-of-freedom model of response to a downward shock (4-Hz nominal frequency; $0.315 \mathrm{~ms}^{-1.75}$ ). Data from 20 subjects. 
High magnitude upward

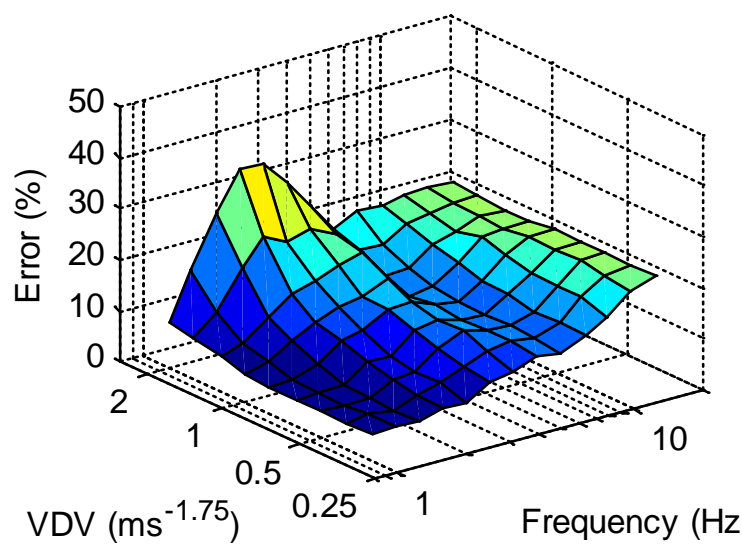

Low magnitude upward

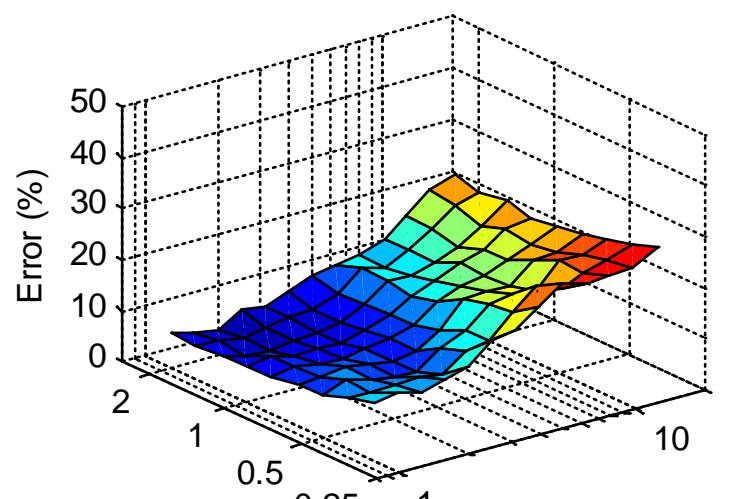

$\operatorname{VDV}\left(\mathrm{ms}^{-1.75}\right) \quad 0.25 \quad 1 \quad$ Frequency $(\mathrm{Hz})$
High magnitude downward

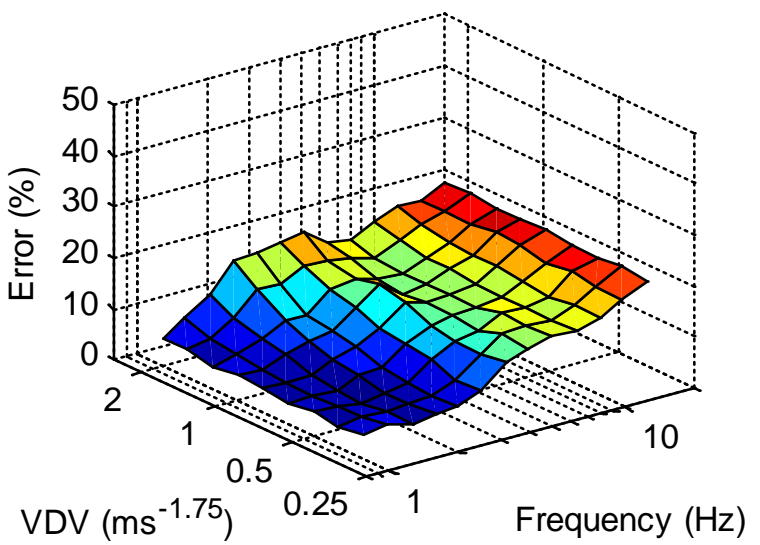

Low magnitude downward

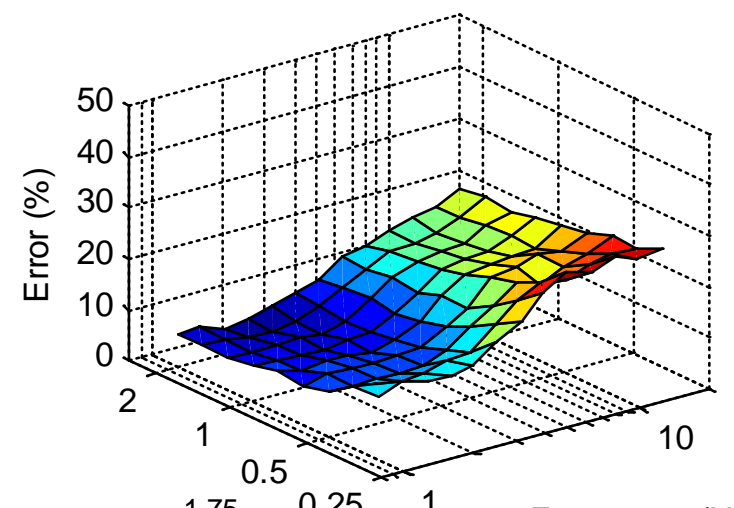

$\operatorname{VDV}\left(\mathrm{ms}^{-1.75}\right) \quad 0.25 \quad 1 \quad$ Frequency $(\mathrm{Hz})$

Figure 12 Median error between the measured acceleration waveform and the fitted acceleration waveform (i.e., $\delta_{a}$ ) at each frequency with higher magnitude shocks (upper figures) and lower magnitude shocks (lower figures) and for upward shocks (left figures) and downward shocks (right figures) using a single degree-of-freedom model with stiffness and damping optimised in the frequency domain with $0.1 \mathrm{~ms}^{-2}$ r.m.s. random vibration. 

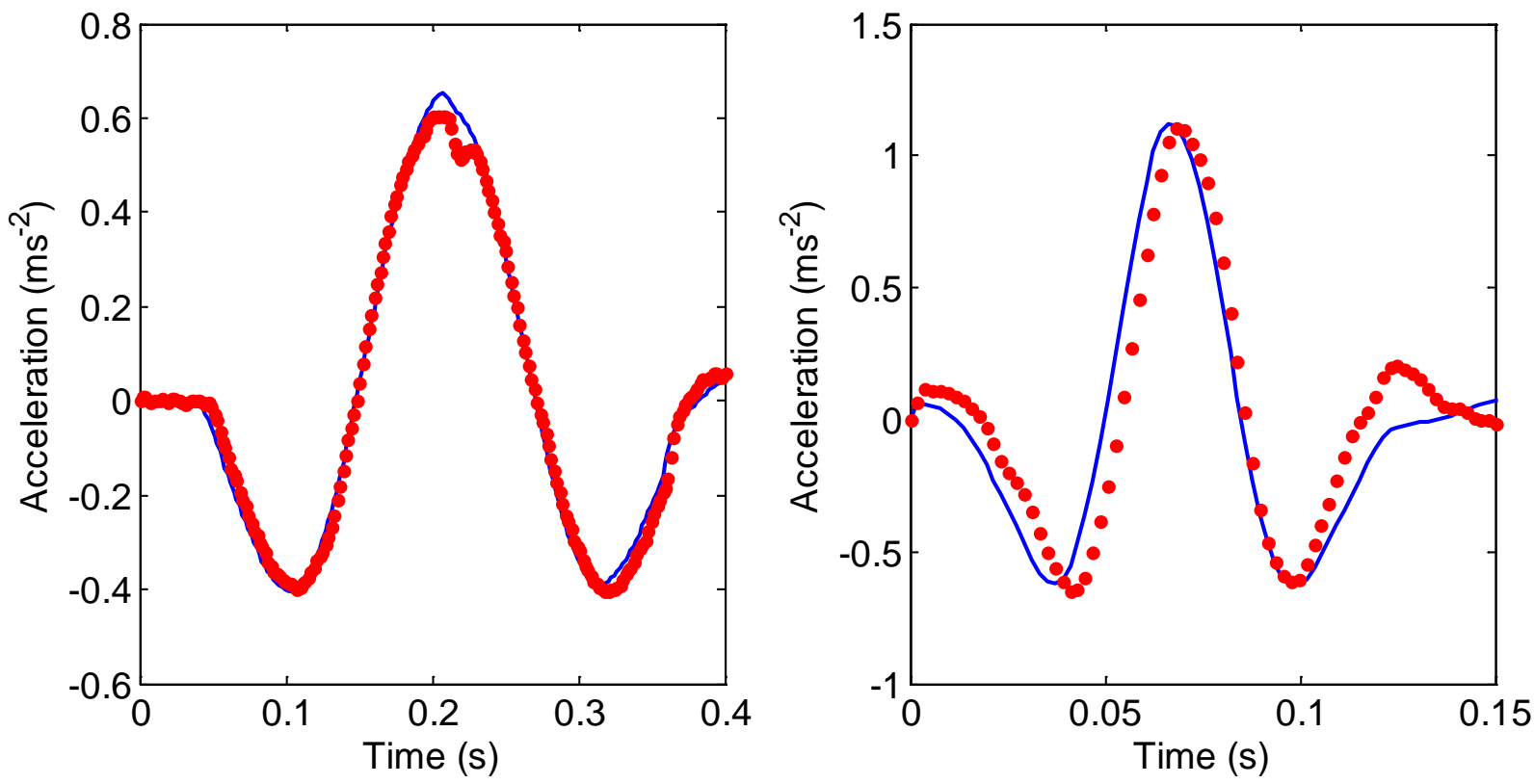

Figure 13 Comparison of fitted acceleration waveforms and measured acceleration waveforms (left: $4 \mathrm{~Hz}$; right: $16 \mathrm{~Hz}$ ). Data from one subject with low magnitude downward shocks. Output acceleration waveforms ( $\longrightarrow$ ) and fitted

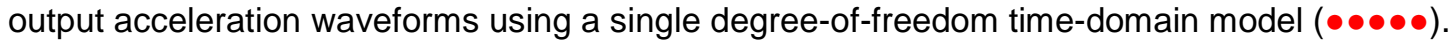

